\title{
Do horizontal saccadic eye movements increase interhemispheric coherence? Investigation of a hypothesized neural mechanism underlying EMDR
}

\author{
Zoe Samara' ${ }^{1,2 *}$, Bernet M. Elzinga ${ }^{1,2}$, Heleen A. Slagter ${ }^{3}$ and Sander Nieuwenhuis ${ }^{2,4}$ \\ 1 Clinical, Health and Neuropsychology Unit, Institute for Psychological Research, Leiden University, Leiden, Netherlands \\ 2 Leiden Institute for Brain and Cognition, Leiden University, Leiden, Netherlands \\ ${ }^{3}$ Brain and Cognition Unit, Department of Psychology, University of Amsterdam, Amsterdam, Netherlands \\ ${ }^{4}$ Cognitive Psychology Unit, Institute for Psychological Research, Leiden University, Leiden, Netherlands
}

\section{Edited by:}

Zafiris J. Daskalakis,

University of Toronto, Canada

Reviewed by:

Arielle D. Stanford,

Columbia University, USA

Paul Croarkin, University of Texas

Southwestern Medical Center, USA

*Correspondence:

Zoe Samara, Department of

Neuropsychology and

Psychopharmacology, Faculty of

Psychology and Neuroscience,

Maastricht University, PO Box 616,

6200 MD Maastricht, Netherlands.

e-mail: z.samara@maastrichtuniversity.nl
Series of horizontal saccadic eye movements (EMs) are known to improve episodic memory retrieval in healthy adults and to facilitate the processing of traumatic memories in eye movement desensitization and reprocessing (EMDR) therapy. Several authors have proposed that EMs achieve these effects by increasing the functional connectivity of the two brain hemispheres, but direct evidence for this proposal is lacking. The aim of this study was to investigate whether memory enhancement following bilateral EMs is associated with increased interhemispheric coherence in the electroencephalogram (EEG). Fourteen healthy young adults were asked to freely recall lists of studied neutral and emotional words after a series of bilateral EMs and a control procedure. Baseline EEG activity was recorded before and after the EM and control procedures. Phase and amplitude coherence between bilaterally homologous brain areas were calculated for six frequency bands and electrode pairs across the entire scalp. Behavioral analyses showed that participants recalled more emotional (but not neutral) words following the EM procedure than following the control procedure. However, the EEG analyses indicated no evidence that the EMs altered participants' interhemispheric coherence or that improvements in recall were correlated with such changes in coherence. These findings cast doubt on the interhemispheric interaction hypothesis, and therefore may have important implications for future research on the neurobiological mechanism underlying EMDR.

Keywords: horizontal, saccadic, eye, movements, coherence, interhemispheric, emotional, EMDR

\section{INTRODUCTION}

Posttraumatic stress disorder (PTSD) is a debilitating condition, characterized by repeated and unwanted reexperiencing of a traumatic event, hyperarousal, and avoidance of stimuli that could serve as reminders for the event. A hallmark symptom of PTSD is memory disturbance, in the form of intrusive memories (flashbacks, nightmares etc). At the same time, PTSD patients tend to have impaired episodic memory, both in the form of overgeneral memory for autobiographical events (see McNally et al., 1995; Schönfeld and Ehlers, 2006) and diminished recall of neutral information (see Elzinga and Bremmer, 2002). Eye movement desensitization and reprocessing (EMDR) therapy, developed by Shapiro (1989), has been established as an effective treatment for patients with PTSD (American Psychiatric Association, 2004; Lamprecht et al., 2004; Bisson et al., 2007; Cukor et al., 2010). EMDR therapy involves processing of the traumatic memories while patients are engaged in bilateral sensory stimulation, most commonly in the form of horizontal saccadic eye movements (EMs; other forms of bilateral stimulation include sound, tapping, or vibration; see ServanSchreiber et al., 2006). This bilateral stimulation is assumed to be instrumental for the alleviation of the memory symptoms of PTSD patients (Montgomery and Ayllon, 1994; Lee and Drummond, 2008; Lilley et al., 2009). Other studies have reported that EMs also decrease the vividness and distress of emotional autobiographical memories in healthy adults (Andrade et al., 1997; Kavanagh et al., 2001; van den Hout et al., 2001, 2010; Barrowcliff et al., 2004; Kemps and Tiggemann, 2007; Gunter and Bodner, 2008; Maxfield et al., 2008; Schubert et al., 2011).

Horizontal saccadic EMs also benefit the recall of neutral autobiographical memories in healthy adults. After a 30-s period of horizontal saccadic EMs immediately prior to retrieval, participants recall more recent and early childhood events (Christman et al., 2003, 2006), and more autobiographical memories cued by neutral and emotional words (Parker and Dagnall, 2010). Moreover, horizontal EMs have also been related to the recall of more neutral words (Christman et al., 2003; Lyle et al., 2008a; Parker et al., 2008), fewer critical lures in the verbal converging semantic associates (DRM) paradigm (Christman et al., 2004; Parker and Dagnall, 2007), more details in a visual event narrative (Parker et al., 2009), and more landmark shape and location information (Brunyé et al., 2009). In these studies, the EM procedure was designed to be similar to that used in EMDR: participants watched a dot that alternately appeared on the left and right side of the screen, changing position twice per second. This procedure was compared with a control condition, in most studies consisting of vertical saccadic EMs or a centrally presented dot changing color twice per second. 
Although the effects of EMs on (sub)traumatic autobiographical memories and neutral episodic memories seem hard to reconcile, researchers have proposed a similar neural basis for these effects. For example, Servan-Schreiber (2000) has suggested that the EMs involved in the EMDR procedure may benefit memory by increasing the synchronization of the hemispheres and their functional connectivity (see Bergmann, 1998, for a similar account). He hypothesized that bilateral saccadic EMs may "induce a background of synchronous neural activity across cerebral hemispheres" which is supposed to "contribute to the reintegration of dissociated aspects of memories by re-establishing a synchrony between functionally disconnected areas" (see p. 18). As such, EMDR may promote the integration of the traumatic material with verbal representations of the larger context of the patient's life. Likewise, Propper and Christman (2008) conclude, on the basis of a literature review, that the improvements in episodic memory observed after bilateral EMs reflect increased interhemispheric interaction. Evidence that interhemispheric interaction is an important component of episodic memory is provided by imaging studies showing asymmetric lateralization of prefrontal activity during episodic encoding (i.e., left side of the brain) vs. retrieval (i.e., more right/bilateral activation; Habib et al., 2003). Other indirect evidence comes from visual half-field studies (Christman and Propper, 2001) and studies showing impaired episodic memory in strongly right-handed individuals (Christman et al., 2004; Propper et al., 2005; Lyle et al., 2008b).

Despite these proposals, there is only limited and indirect evidence that the effects of EMs on memory are mediated by increased interhemispheric interaction. First, one study has shown that memory performance of strongly right-handed individuals increased after EMs almost to the level of mixed-handed individuals, whose performance did not improve (Lyle et al., 2008a). This finding suggests that EMs allow strongly right-handers to temporarily compensate for their reduced functional hemispheric connectivity compared to mixed handers, whose differential brain lateralization calls for enhanced flow of information between the hemispheres (mediated by their enhanced corpus callosum size; e.g., Witelson and Goldsmith, 1991). Second, interhemispheric electroencephalography (EEG) coherence (a measure of hemispheric functional connectivity) increases during REM sleep (Dummermuth and Lehman, 1981; Barcaro et al., 1989) and this increase has been specifically linked to the occurrence of horizontal EMs during REM phases (Dionne, 1986). And third, Bakan and Svorad (1969) have found that lateral EMs lead to a sustained increase in the activation of the contralateral hemisphere. Thus, repeated leftright EMs presumably result in simultaneous activation of both hemispheres, which is assumed to foster interhemispheric communication (Propper and Christman, 2008).

A recent study has tried to address the interhemispheric interaction hypothesis more directly using EEG. Propper et al. (2007) measured frontal interhemispheric gamma EEG coherence after $30 \mathrm{~s}$ of bilateral saccadic EMs. Interhemispheric coherence is thought to reflect long-range functional connectivity between corresponding brain regions in the two hemispheres, mediated primarily via the corpus callosum (Knyazeva et al., 1999). Somewhat surprisingly, Propper et al. (2007) found that the EMs reduced interhemispheric coherence. Unfortunately, however, this study had several methodological limitations: it involved a (less powerful) between-subjects design; it did not include a memory task, so changes in EEG coherence could not be associated with changes in memory performance; coherence estimates were calculated on two 1-s epochs, which is too short for a good estimate, and therefore likely to yield a noisy measure of coherence; and the EEG signal was recorded from only one pair of (prefrontal) electrodes.

The main aim of this EEG study was to test the interhemispheric interaction hypothesis, while addressing the limitations of the Propper et al. (2007) study. Fourteen healthy young adults came to our lab twice and on both occasions studied a list of neutral and emotional words. After a retention period they were asked to recall as many of the words as possible. Prior to this retrieval phase participants engaged in $30 \mathrm{~s}$ of horizontal saccadic EMs (in one session) or a control procedure (in the other session). Immediately before and after this procedure, we collected baseline EEG data from 31 electrodes covering the entire scalp. To investigate the effects of horizontal EMs on interhemispheric communication we conducted EEG analyses to examine EM-related changes in the synchronization of neural activity - or coherence - between symmetric scalp regions.

According to the interhemispheric interaction hypothesis, interhemispheric coherence should increase (or alter) following the saccadic EM procedure compared to the control procedure. Although changes in interhemispheric coherence were examined for all electrode sites, given the established role of the prefrontal cortices in episodic retrieval, we specifically predicted EM-related changes in interhemispheric coherence over prefrontal brain regions. In addition, we hypothesized that if interhemispheric interaction is an important component of episodic memory, inter-individual differences in interhemispheric coherence should correlate with performance in the free recall task. Although the recall of neutral experimental stimuli after horizontal saccadic EMs has been widely investigated, no studies have yet examined the effects of EMs on memory for emotional material. In this study, participants were therefore presented with both neutral and emotional words in order to examine whether the EMs have a similar impact on emotional vs. neutral word recall.

\section{MATERIALS AND METHODS PARTICIPANTS}

Fourteen female students at Leiden University $(M$ age $=20.9$, $\mathrm{SD}=3.08)$ participated in the study for course credit or $€ 15$. All participants were native speakers of Dutch and had normal or corrected-to-normal vision. Exclusion criteria were: mixed and left-handedness, high levels of psychological distress (as indexed by mean score $>149$ on the Symptoms Checklist 90 (SCL90, Arrindell and Ettema, 1986), neurological symptoms, head-trauma history, and substance use/addiction. Handedness was assessed by the Edinburgh Handedness Inventory (Oldfield, 1971). Participants scoring +7.5 and below were classified as mixed-handed and excluded, whereas those scoring +8.0 and above were classified as strongly right-handed and included $(M$ score $=98.3, \mathrm{SD}=5.77)$.

\section{STIMULI}

In the EM condition, a single black dot with a diameter of approximately $4^{\circ}$ of visual angle appeared sequentially on the left and right portion of the display (at $27^{\circ}$ apart) for $30 \mathrm{~s}$. The dot changed 
position every $500 \mathrm{~ms}$, leading to two saccadic EMs per second (see Christman et al., 2003; Parker and Dagnall, 2007; Lyle et al., 2008a for similar procedures). In the control condition, a colored circle of the same diameter as the black dot was presented centrally. The circle changed color twice per second, alternating between green and red. The control condition was adopted from previous studies (Christman et al., 2004, 2006) and was preferred over a "do-anything" 30-s period in order to avoid spontaneous EMs mimicking horizontal bilateral saccades.

For encoding, words were presented in black font in the center of a white screen. The word stimuli for encoding consisted of 144 common Dutch words selected from Hermans and De Houwer's (1994) list of 740 Dutch words rated for their subjective familiarity and affectivity (see Table 1 for a sample of the stimuli). The words were divided in two sets of 72 words that were matched in length $(M=7.3, \mathrm{SD}=1.8)$ and frequency $(M=781.2, \mathrm{SD}=1053.6)$ that were counterbalanced across the two conditions. There were no list effects on the number of recalled words [ $t(13)=0.7, p=0.47]$.

\section{PROCEDURE}

The study was approved by the ethical committee of the Leiden University Medical Centre and carried out in accordance with the latest version of the Declaration of Helsinki.

All participants were tested in the EM condition and the control condition, in two separate 1-h sessions, 1 week apart. The order of conditions was counterbalanced across participants. At the start of

Table 1 | Sample of the Dutch words included in the study lists with their English translation.

Example memory stimuli

\begin{tabular}{lllll}
\hline & \multicolumn{2}{c}{ List $\mathbf{1}$} & & \multicolumn{2}{c}{ List 2 } \\
\cline { 1 - 1 } Dutch & Translation & & Dutch & Translation \\
\cline { 1 - 1 } NEUTRAL WORDS & & & & \\
Appel & Apple & & Legpuzzel & Jigsaw \\
Grammatica & Grammar & & Aankleden & To dress \\
Document & Document & & Handtas & Handbag \\
Driehoek & Triangle & & Bestek & Cutlery \\
Elleboog & Elbow & & Centimeter & Centimeter \\
Portret & Portrait & & Dossier & File \\
Potlood & Pencil & & Etiket & Label \\
Meubel & Furniture & & Badkamer & Bathroom \\
Supermarkt & Supermarket & & Deurbel & Doorbell \\
Mechanisch & Mechanical & & Ademhaling & Breathing \\
EMOTIONAL WORDS & & & \\
Sadist & Sadist & & Afgrijzen & Horror \\
Tranen & Tears & & Tumor & Tumor \\
Fobie & Phobia & Bloed & Blood \\
Trauma & Trauma & Mislukking & Failure \\
Verraad & Betrayal & Verdriet & Grief \\
Inbraak & Burglary & Woede & Rage \\
Kanker & Cancer & & Gevaar & Danger \\
Armoede & Poverty & & Sterven & To die \\
Bom & Bomb & & Pistool & Pistol \\
Moord & Murder & & Haat & Hate \\
& & & &
\end{tabular}

each session participants were seated in front of a computer monitor. Informed consent of the participants was obtained after the nature of the procedures had been explained. Participants were given explicit instructions to "pay attention to the words as this is a memory test and you will be asked about them later." Then, participants studied one word list, preceded by three additional (neutral) words that served as buffer against the primacy effect, and followed by three additional (neutral) words to guard against recency effects. The study phase began with a fixation cross, followed by a sequence of words, each presented for $2000 \mathrm{~ms}$. After the study phase, there was a 30-min interval during which participants were prepared for the EEG recording phase. During this interval, participants watched a neutral documentary, serving as a filler task. Then, pre-condition baseline EEG data were collected during a 4-min period ${ }^{1}$ in which participants alternated each minute between keeping their eyes open and closed.

After that, participants engaged in either the EM procedure or the control procedure. During this phase, the experimenter closely monitored participants' compliance with the instructions. Participants' compliance with instruction was also ensured by recording their saccadic electro-oculograms (EOG) during this phase. In the EM condition participants were instructed to focus on the dot appearing on the screen and follow it by moving their eyes and not their head. In the control condition, participants were told to keep their eyes at the colored dot. Immediately after this procedure, post-condition baseline EEG data were collected during a 4-min period, using the previous scheme. After the baseline EEG recordings, participants were asked to write on a piece of paper as many words as they could remember from the study list within $5 \mathrm{~min}$. At the end of the experiment participants were paid and debriefed.

\section{EEG RECORDING}

Electroencephalography was recorded at $512 \mathrm{~Hz}$ from $31 \mathrm{Ag} / \mathrm{AgCl}$ electrodes mounted in an elastic cap according to the International 10-20 system, and from the left and right mastoids using a 64-channel Biosemi active electrode recording system. Individual sensors were adjusted and impedances were kept lower than $25 \mathrm{k} \Omega$. Two additional electrodes (Common Mode Sense, CMS; and Driven Right Leg, DRL) were used as reference and ground. The horizontal and vertical EOG were measured using bipolar recordings from electrodes placed approximately $1 \mathrm{~cm}$ lateral of the outer canthi of the two eyes and from electrodes placed approximately $1 \mathrm{~cm}$ above and below participants' right eye.

\section{EEG DATA ANALYSIS}

EEGLAB (Delorme and Makeig, 2004) and Matlab (Mathworks, Natick, MA, USA) were used for off-line EEG data processing. After applying a $50-\mathrm{Hz}$ notch filter, data were visually inspected and cleaned of large movement-related artifacts and bad segments. Consistently bad channels in a given session were interpolated using a spherical spline algorithm. ICA was then used to remove ocular and muscle artifacts (Jung et al., 2000). Data were segmented into 4096-ms epochs and visually inspected in a second round for remaining artifacts, which were manually removed. The mean number of artifact-free epochs used in the coherence analyses was 19.9 (i.e., $81 \mathrm{~s}$ ).

${ }^{1}$ For two participants only $2 \mathrm{~min}$ of data were available. 
To investigate the hypothesis that horizontal EMs produce changes in interhemispheric communication, we computed between-channel phase and amplitude coherence, using the baseline EEG data obtained right before and after the EMs and the control condition and examined EM-specific changes in interhemispheric coherence. To this end, we first re-referenced the artifact-free epoched data to the average of all channels (i.e., average reference) or, in additional analyses, subtracted from each channel the weighted average of the signals occurring at the immediately surrounding electrodes (i.e., Laplacian reference; Essl and Rappelsberger, 1998). It is well known that choice of reference can influence the outcome of coherence analyses (Nunez and Srinivasan, 2006) ${ }^{2}$. For this reason, we ran our coherence and correlation analyses on both average-referenced and Laplacian-referenced data. Cross-channel baseline phase and amplitude coherence were estimated separately for 12 interhemispheric electrode pairs (Fp1-Fp2, F3-F4, FC3-FC4, C3-C4, CP3CP4, P3-P4, O1-O2, F7-F8, FT7-FT8, T7-T8, TP7-TP8, P7-P8), for six standard frequency bands: $\theta(4-7 \mathrm{~Hz}), \alpha(8-13 \mathrm{~Hz})$, lower $\beta$ (14-21 Hz), higher $\beta(22-33 \mathrm{~Hz})$, lower $\gamma(36-44 \mathrm{~Hz})$, and higher $\gamma(44-90 \mathrm{~Hz})$ using Hanning-tapered, zero-padded fast Fourier transforms (Delorme and Makeig, 2004). Phase coherence for two signals $a$ and $b$ was defined as:

$\mathrm{COH}^{a, b}(f, t)=\frac{1}{n} \sum_{k=1}^{n} \frac{F_{k}^{a}(f, t) F_{k}^{b}(f, t)^{*}}{\left|F_{k}^{a}(f, t) F_{k}^{b}(f, t)\right|}$

where $n$ is the number of trials and $*$ denotes the complex conjugation. $F_{k}(f, t)$ represents the spectral estimates for a trial $k$, at time $t$ and frequency $f$, for a given signal. Thus, the Fourier transform of one signal was multiplied with the complex conjugate of another signal. The resulting cross-power spectrum was then normalized by power of the given frequency $f$ (Delorme and Makeig, 2004). The magnitude of cross-channel coherence varies between 0 and 1 , a value of 0 indicating a complete absence of synchronization at the given frequency $f$ in the time window centered on $t$, and 1 indicating perfect synchronization. Amplitude coherence was computed by correlating the spectral estimates for trial $k$ of signals $a$ and $b$ at time $t$ and frequency $f$ (Delorme and Makeig, 2004).

Changes in post vs. pre baseline phase and amplitude coherence between conditions were then compared using paired-samples $t$-tests. In addition, in order to examine whether changes in interhemispheric coherence were associated with changes in memory performance, we ran correlation analyses between differences in changes in coherence between conditions and the corresponding differences in changes in the number of freely recalled words, separately for the neutral and emotional stimuli. To correct for multiple comparisons, a significance criterion of $\alpha<0.004$ (i.e., $\alpha=0.05 / 12$ electrode pairs) was adopted. Since our main prediction was that horizontal EM would selectively affect interhemispheric communication, we focused on differences in interhemispheric coherence between the EM vs. control condition and, if observed, examined whether these were driven by changes in post vs. pre baseline coher-

${ }^{2}$ While average reference provides reasonable semi-quantitative estimates of largescale neocortical source coherence, it may overestimate coherence due to volume conduction. Conversely, the Laplacian method removes most reference electrode and volume conduction distortion, but may underestimate coherence by spatial filtering. ence in the EM condition, the control condition, or both. Only those differences in coherence between the EM and control conditions that were also accompanied by a significant change in coherence in the EM condition are reported here (but see Tables 2 and 3 for an overview of all significant effects).

\section{RESULTS}

\section{BEHAVIORAL DATA}

Paired-samples $t$-tests (one-tailed) indicated that in the EM condition, participants recalled more emotional words $(M=5.9$, $\mathrm{SD}=2.5)$ than in the control condition $(M=4.5, \mathrm{SD}=3.0)$, $t(13)=2.1, p=0.026$, Cohen's $d=0.51$, indicating a medium effect). There was no difference between the number of recalled neutral words in the EM condition $(M=4.1, \mathrm{SD}=2.4)$ and the control condition $(M=4.0, \mathrm{SD}=2.9), t(13)=0.1, p=0.47$ (see Figure 1).

\section{EEG DATA}

Based on the interhemispheric interaction hypothesis, we predicted increased interhemispheric phase and/or amplitude coherence as a result of participants' engagement in the EM condition, reflecting altered functional connectivity between the hemispheres. Figures 2A,B show changes in baseline interhemispheric phase and amplitude coherence, respectively, as a function of condition (EM, control). One lateral frontal electrode pair, FT7-FT8, displayed a significant difference in post vs. pre alpha-band amplitude coherence in the EM vs. the control condition, $t(13)=5.0, p=0.0002$ (Laplacian-referenced data). Post hoc paired $t$-tests revealed that this difference was driven by significant changes in amplitude coherence in both the EM and the control condition. Specifically, amplitude coherence decreased after horizontal EMs, $t(13)=3.5, p=0.004$ while amplitude coherence increased after the control condition, $t(13)=3.6, p=0.003$. Based on the interhemispheric interaction hypothesis, one would predict that interhemispheric communication is selectively altered by horizontal EMs, rendering this effect, which was driven by both conditions, difficult to interpret. No other EM-related changes in interhemispheric coherence were observed in the average-referenced and the Laplacian-referenced data, all $p s>0.004$. Our analyses did reveal other significant changes in coherence in the EM condition or in the control condition that did not reliably differ between the two conditions, and hence do not reflect selective effects of horizontal EMs on interhemispheric communication. These results are reported in Tables 2 and 3 . To exclude the possibility that we missed subtle changes in coherence due to the stringency of the corrected $\alpha$ value used, we also examined the results using $\alpha \leq 0.01$ as significance criterion. No additional significant differences in interhemispheric coherence driven by the EM condition were found for any of the six frequency bands.

We next performed correlational analyses to examine whether individual differences in EM-related changes in interhemispheric connectivity were predictive of changes in memory performance. No relationship was observed across participants between EM-related changes in phase and amplitude coherence and corresponding changes in the recall of neutral or emotional words (all ps > 0.004; see also Tables 2 and 3). With a less stringent threshold of $p<0.01$, similarly, no changes in coherence driven by the EM condition were associated with changes in the recall of neutral or emotional words. 
Table 2 | Summary of significant changes in phase coherence for the average and the Laplacian reference scheme (significance threshold adjusted for number of electrode pairs).

\begin{tabular}{|c|c|c|c|c|c|c|c|}
\hline Reference & \multicolumn{7}{|c|}{ Phase coherence, $p<0.004$} \\
\hline \multirow[t]{7}{*}{ Average } & t-test EM-C & & & & & & \\
\hline & t-test EM & & & P7-P8 & & CP3-CP4 & \\
\hline & Corr EM-C Neut & & & & & & \\
\hline & Corr EM-C Emo & & & & & & \\
\hline & Corr EM All & & & & & & \\
\hline & Corr EM Neut & & & & & & \\
\hline & Corr EM Emo & & & & & & \\
\hline \multirow[t]{11}{*}{ Laplacian } & t-test EM-C & & & & & & \\
\hline & t-test EM & & & & & & \\
\hline & t-test C & & & & P7-P8 & & \\
\hline & Corr EM-C All & & & F3-F4 & & & \\
\hline & Corr EM-C Neut & & & & & & \\
\hline & Corr EM-C Emo & & & & & & \\
\hline & Corr EM All & & & & & & \\
\hline & Corr EM Neut & & & & & & \\
\hline & Corr EM Emo & & & & & & \\
\hline & Corr C All & & & & & & \\
\hline & Corr C Neut & & & & $\mathrm{C} 3-\mathrm{C} 4$ & & \\
\hline
\end{tabular}

Corr, correlation; EM, eye movement condition; C, control condition; Neut, neutral words; Emo, emotional words.

Propper et al. (2007) found that the EM procedure reduced interhemispheric coherence in the gamma band $(35-54 \mathrm{~Hz})$ at anterior frontal electrodes (FP1-FP2). They used a between-subjects design and a measure of coherence that depends mostly on the consistency of phase differences between electrodes (Nunez et al., 1997; Nunez and Srinivasan, 2006). We therefore examined whether we could replicate their findings using the same coherence measure and a between-subject approach ${ }^{3}$, by including only data from the first session. Participants were divided into two groups based on the condition (EM or control) they participated in during this session. Gamma-band coherence between channels FP1 and FP2 was computed on the artifact-free epoched data, separately for each participant and the pre- and post-condition baseline EEG data sets. In contrast to Propper et al. (2007), we did not find a difference between groups in changes in interhemispheric frontal gamma coherence, $t(12)=0.04, p=1$ for the average-referenced data and $t(12)=0.67, p=0.5$ for the Laplacian-referenced data.

${ }^{3}$ The same analysis was also performed using a within-subjects approach, revealing no differences in frontal gamma coherence in FP1 and FP2 pair between the two conditions; $t(13)=0.40, p=0.7$ for the average-referenced data and $t(13)=0.60$, $p=0.6$ for the Laplacian-referenced data.

\section{DISCUSSION}

The main aim of this study was to test a key prediction of the interhemispheric communication hypothesis of EMDR: the hypothesis that bilateral horizontal EMs increase the synchronization between the hemispheres, therewith facilitating episodic recall (Servan-Schreiber, 2000; Propper and Christman, 2008). We used EEG to examine whether engagement for $30 \mathrm{~s}$ in horizontal saccadic EMs resulted in increased (or altered) interhemispheric connectivity between symmetric scalp locations. Our data yielded very little evidence for this hypothesis: interhemispheric phase and amplitude EEG coherence among 12 channel pairs and across six frequency bands were not selectively affected by the EM procedure. As discussed below, the only exception concerned a pair of lateral frontal electrodes that showed a decrease in amplitude coherence in the alpha band $(8-13 \mathrm{~Hz})$, but this effect was driven by changes in alpha-amplitude coherence in both the EM and the control condition. We also investigated whether EM-related changes in interhemispheric connectivity predicted corresponding changes in episodic memory performance. Our data did not confirm this prediction either. Therefore, our results do not support the idea that the beneficial effect of EMs on memory retrieval is mediated by changes in interhemispheric interaction between homologous areas at the cortical level. 
Table 3 | Summary of significant changes in amplitude coherence for the average and the Laplacian reference scheme (significance threshold adjusted for number of electrode pairs).

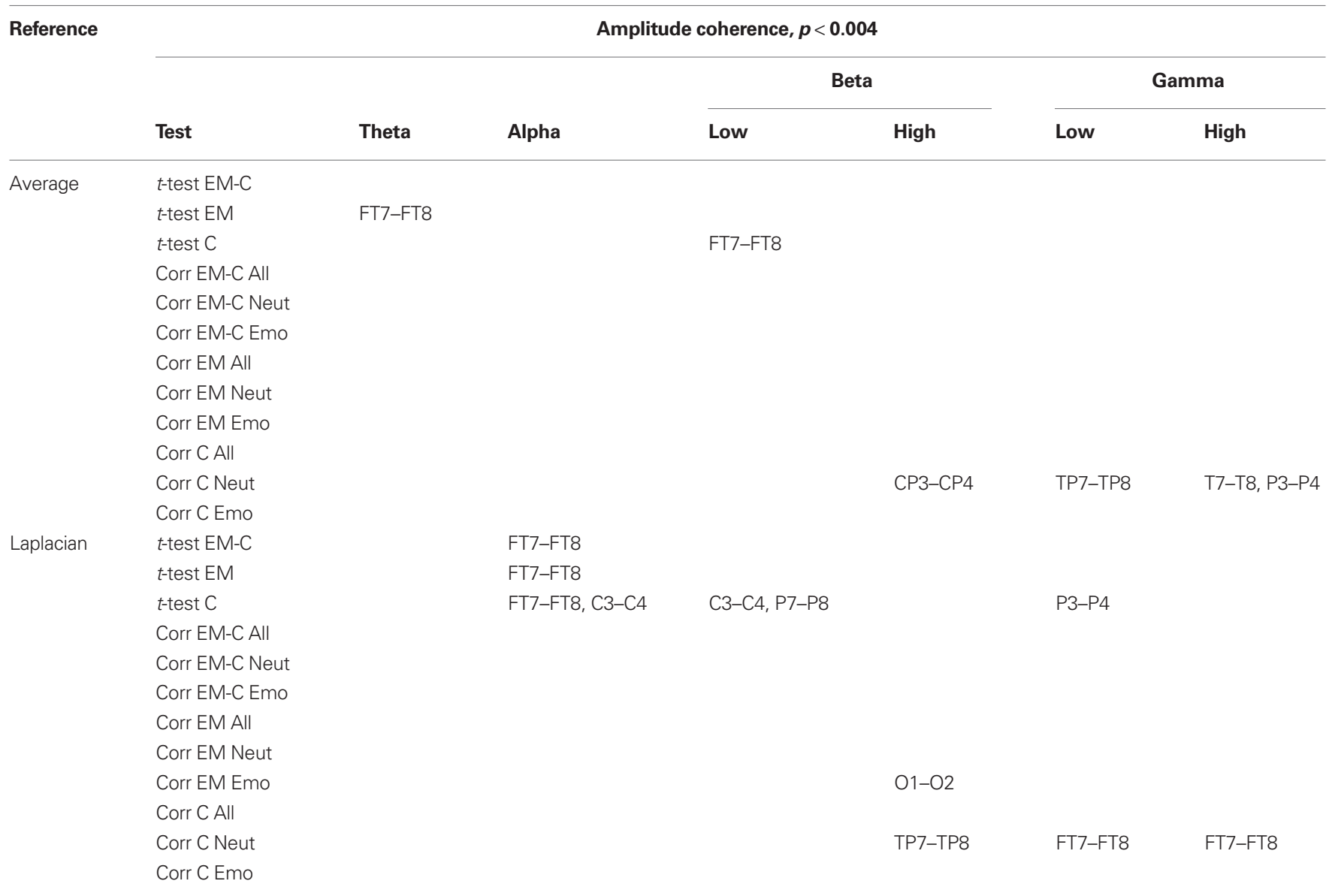

Corr, correlation; EM, eye movement condition; C, control condition; Neut, neutral words; Emo, emotional words.

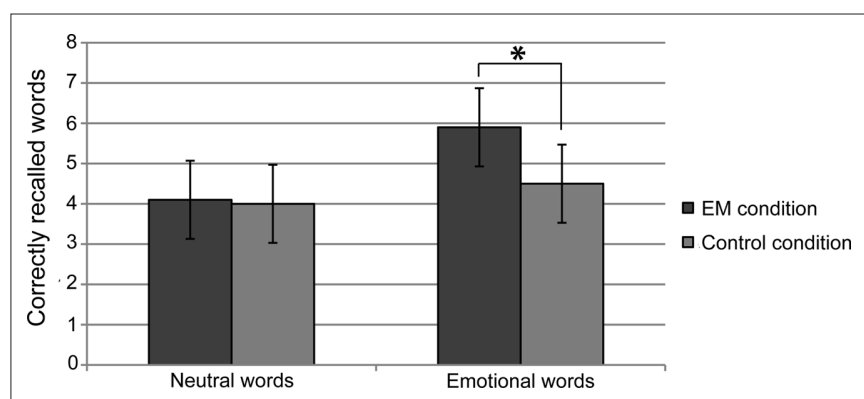

FIGURE 1 | Mean number of freely recalled neutral and emotional words in the eye movement (EM) condition and the control condition. Error bars indicate the $97 \%$ confidence interval.

While research has convincingly linked alpha power to the excitability or activation of underlying cortical tissue (e.g., Pfurtscheller, 2001; Klimesch et al., 2007), less is known about the functional significance of inter-regional alpha synchronization during resting-state conditions. Alpha coherence between homologous frontal sites has been shown to be state dependent in some studies. For example, alpha coherence between frontal sites was found to become weaker from wakefulness to drowsiness to REM sleep (Cantero et al., 1999), and to be reduced during a cognitive task vs. resting state (Nunez, 2000). The here observed EM-related decrease in alpha-band coherence over frontal sites might thus reflect changes in brain state related to decreased arousal or cognitive processing. Yet, it is unclear how decreased arousal or cognitive processing functionally would lead to memory enhancement. Furthermore, several observations suggest caution in interpreting this effect: First, as mentioned above, alpha-band amplitude coherence between frontal electrodes was not only affected in the EM condition; the control condition showed a significant increase in coherence. Second, there was no relationship between EM-related changes in alpha-band amplitude coherence and corresponding changes in memory task performance. Third and lastly, amplitude coherence is much more sensitive to artifactual changes in baseline EEG, for example, residual eye movement-related activity, than phase coherence. Therefore, the current finding should be interpreted with caution, and future studies are necessary to determine its robustness.

Our main findings contrast with the findings of Propper et al. (2007), who found that $30 \mathrm{~s}$ of bilateral saccadic EMs led to a decrease in interhemispheric coherence in the gamma band. Our 


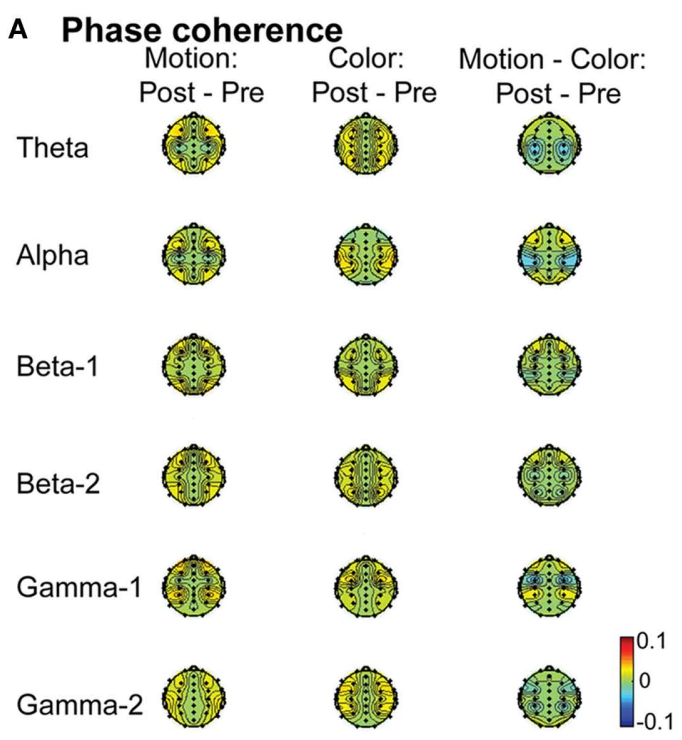

FIGURE 2 | (A) Group average difference topographic head maps of phase coherence values in theta $(4-7 \mathrm{~Hz})$, alpha $(8-13 \mathrm{~Hz})$, lower beta $(14-21 \mathrm{~Hz})$, higher beta (22-33 Hz), lower gamma (36-44 Hz), and higher gamma (44-90 Hz) frequency bands averaged across the 4-min baseline recording periods before and after the EM vs. the control manipulation (averagereference data). The magnitude of cross-channel coherence varies between 0 and 1 , with 0 indicating a complete absence of synchronization and 1 indicating perfect synchronization. Colors here denote changes in coherence, with yellow-red (positive values) denoting increased coherence, and greenblue (negative values) denoting decreased coherence. (B) Group average

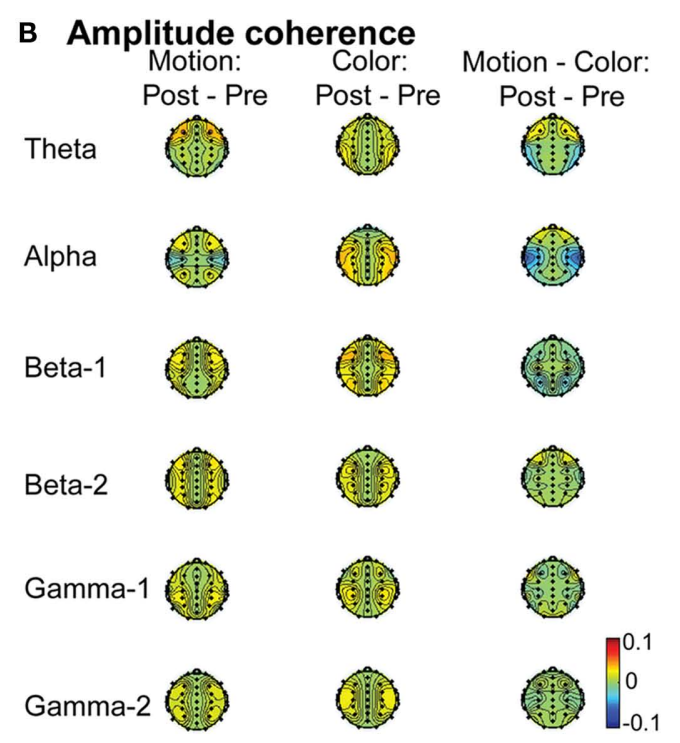

difference topographic head maps of amplitude coherence values in theta (4-7 Hz), alpha $(8-13 \mathrm{~Hz})$, lower beta $(14-21 \mathrm{~Hz})$, higher beta $(22-33 \mathrm{~Hz})$, lower gamma (36-44 Hz), and higher gamma (44-90 Hz) frequency bands averaged across the 4-min baseline recording periods before and after the EM vs. the control manipulation (average-reference data). The magnitude of cross-channel coherence varies between 0 and 1, with 0 indicating a complete absence of synchronization and 1 indicating perfect synchronization. Colors here denote changes in coherence, with yellow-red (positive values) denoting increased coherence, and green-blue (negative values) denoting decreased coherence. study addressed some important limitations of the study by Propper et al. (2007). First, we used a within-subjects design, which allows a more powerful comparison between task conditions. Second, we calculated both phase and amplitude coherence, for twelve electrode pairs covering the entire scalp, and we based our coherence estimates on multiple 4-s epochs. Finally, we computed coherence for both average-referenced and Laplacian-referenced data. Given these methodological strengths, it is unlikely that we missed any evidence of altered interhemispheric coherence (present in the EEG signal). Of further importance, a post hoc analysis that used the same coherence measure and a between-subject approach, as Propper et al. (2007) did, but was based on multiple 4-s epochs, did not replicate their previous finding. Therefore, our results are inconsistent with the notion that horizontal EMs alter the functional connectivity of the hemispheres.

Although experimental studies have repeatedly demonstrated the beneficial effects of EMs on memory for neutral stimuli, our study is the first to demonstrate that $30 \mathrm{~s}$ of bilateral saccadic EMs enhance the episodic retrieval of non-traumatic emotional stimuli in healthy adults. In contrast, we did not replicate previous findings of enhanced recall of neutral stimuli (Christman et al., 2003; Lyle et al., 2008a; Parker et al., 2008). This may be due to a design difference between our study and previous studies: in most previous studies the EM manipulation immediately preceded the recall test, whereas in our study the EM manipulation and recall test (which lasted $\sim 5 \mathrm{~min}$ ) were separated by the post-condition baseline EEG measurement, which lasted $4 \mathrm{~min}$. The time frame of EM-related effects on memory is still unknown. Hence, it is possible that the effects do not last longer than 4-9 min, which would be too short to affect the retrieval of the neutral words in our paradigm. Moreover, the EM-related effects might have been too weak to improve recall of neutral stimuli but strong enough to improve recall of emotional words. The only indication for the duration of the effects of EMs we found was in a study of Shobe et al. (2009), who investigated the effect of bilateral EMs on dimensions of creativity, showing that the effect of EMs on originality lasted up to 7-9 $\mathrm{min}$, while the effect on categorical distinctiveness, another creativity dimension, lasted 4-6 min. However, it is unclear whether these time estimates generalize to effects on memory.

What are the implications of this study for the mechanism underlying EMDR? At the behavioral level, the finding that EMs enhance the recall of non-traumatic emotional stimuli raises important questions regarding the nature of the changes in memory effected by the EMs and the role of these changes in clinical improvement. Currently, we lack a sufficient understanding of the enhancement of normal neutral and emotional recall following horizontal saccadic EMs vis-à-vis the reduction in vividness and distress associated with emotional autobiographical memories. Furthermore, it is not yet clear how these changes are related to the EMDR-related reduction of symptoms associated with traumatic memories, such as intrusions and flashbacks in patients with PTSD. 
At the neurobiological level, the present results provide an important challenge for the proposal of Christman and Propper (2001) that EMs enhance memory through a change in functional connectivity of the right and left prefrontal cortices. Moreover, our findings also suggest that it is unlikely that horizontal EMs produce a significant change in interhemispheric coherence between the other cortical lobes, for example the parietal or temporal lobes, which have been associated with episodic retrieval (Squire et al., 2004; Wagner et al., 2005). Of course, interhemispheric interaction is not limited to homologous areas in the two hemispheres, and our findings do not exclude the possibility that EMs change the interhemispheric interaction between anterior and posterior brain areas or between subcortical areas, thereby influencing memory (cf. Parker and Dagnall, 2010). In a recent report, Lyle and Martin (2010) have found that saccades enhance the accuracy on a lettermatching task when matching targets and probes are presented to the same hemisphere but not when they are presented to different hemispheres. The authors conclude that saccades might enhance attentional processing within each hemisphere without necessarily altering interhemispheric dynamics.

While our study of healthy adults provides an important first step in understanding the EMs effect in relation to EMDR's mechanism of action, we also like to note the limitations of our experimental condition as a model of EMDR therapy for PTSD. Firstly, we tested healthy subjects and not PTSD patients. PTSD patients may be characterized by different neurobiological states and/or cognitive processing styles as a result of persistent PTSD symptoms compared to healthy individuals. Moreover, also the target memories are different in PTSD patients compared to healthy subjects (i.e., traumatic memories of significant arousal and valence that took place months, or even years ago vs. neutral and emotional material of non-traumatic nature that has been encoded 30 min before

\section{REFERENCES}

American Psychiatric Association. (2004). Practice Guidelines for the Treatment of Patients with Acute Stress Disorder and Posttraumatic Stress Disorder. Arlington, VA: American Psychiatric Association.

Andrade, J., Kavanagh, D., and Baddeley, A. (1997). Eye-movements and visual imagery: a working memory approach to the treatment of post-traumatic stress disorder. Br. J. Clin. Psychol.36, 209-223.

Arrindell, W. A., and Ettema, J. H. M. (1986). SCL-90: Handleiding bij een multidimensionele psychopathologieindicator. Lisse: Swets Test Services.

Bakan, P., and Svorad, D. (1969). Resting EEG alpha asymmetry of reflective lateral eye movements. Nature 223, 975-976.

Barcaro, U., Bonanni, E., Denoth, F., Murri, L., Navona, C., and Stefanini, A. (1989). A study of the interhemispheric correlation during sleep in elderly subjects. J. Clin. Neurophysiol. 6, 191-199.

Barrowcliff, A. L., Gray, N. S., Freeman, T. C. A., and MacCulloch, M. J. (2004).
Eye movements reduce the vividness, emotional valence and electrodermal arousal associated with ries. J. Forensic Psychiatry Psychol. 15, 325-345. neurobiology of EMDR. Traumatology 4, 4-16.

Bergmann, U. (2008). The neurobiology of EMDR: exploring the thalamus and neural integration. J. EMDR Pract. Res. 2, 300-314.

Bisson, J. I., Ehlers, A., Mathews, A., Pilling, S., Richards, D., and Turner, S. (2007). Psychological treatments for chronic post-traumatic stress disorder: systematic review and metaanalysis. Br. J. Psychiatry 190, 97-104.

Brunyé, T. T., Mahoney, C. R., Augustyn, J. S., and Taylor, H.A. (2009). Horizontal the retrieval of landmark shape and location information. Brain Cogn. 70, 279-288.

Cantero, J. L., Atienza, M., Salas, R. M., and Gómez, C. (1999). Alpha power modulation during periods with rapid negative autobiographical memo-

Bergmann, U. (1998). Speculations on the saccadic eye movements enhance

retrieval). Secondly, while the number and/or duration of EMs in our study approximates the procedure described in EMDR protocols for administering one set of bilateral stimulation (Shapiro, 2001, 2009; Schubert et al., 2011), EMDR treatment uses multiple sets of EMs during a session and the duration of each individual set is often adjusted to the level of distress associated with the traumatic material. Furthermore, in EMDR sessions the EMs are made while the patient is holding the traumatic memory in mind, and not before its retrieval, as is the case in the laboratory memory paradigms. Taken together, because of these methodological differences, our results may not generalize to patients and/or to sessions in which more sets, or longer-lasting sets, of bilateral stimulation are used. It might well be the case that EMs affect patients' EEG coherence in a different way than that of healthy participants or that $30 \mathrm{~s}$ of EMs are not sufficient to produce a detectable change in EEG coherence.

We acknowledge that our findings should be considered as preliminary evidence, and that they need to be replicated in a larger sample. However, if replicated, the present results shed serious doubts on the interhemispheric interaction hypothesis as the neurobiological mechanism underlying EMDR. In that case, more research is needed to further investigate the neurobiological underpinnings of other neurocognitive models of EMDR, such as the working memory account (Gunter and Bodner, 2008; van den Hout et al., 2010), the thalamo-cortical binding account (Bergmann, 2008), and the possibility that EMDR targets memory through activation of REM-like mechanisms (Hassard, 1996; Stickgold, 2002).

\section{ACKNOWLEDGMENT}

This study was funded by VIDI grants from the Netherlands Organization for Scientific Research (Bernet M. Elzinga and Sander Nieuwenhuis).

oculomotor activity in human REM sleep. Neuroreport 10, 1817-1820.

Christman, S. D., Garvey, K. J., Propper R. E., and Phaneuf, K. A. (2003) Bilateral eye movements enhance the retrieval of episodic memories. Neuropsychology 17, 221-229.

Christman, S. D., and Propper, R. E. (2001). Superior episodic memory is associated with interhemispheric processing. Neuropsychology 15 607-616.

Christman, S. D., Propper, R. E., and Brown, T. J. (2006). Increased interhemispheric interaction is associated with earlier offset of childhood amnesia. Neuropsychology 20, 336-345.

Christman, S. D., Propper, R. E., and Dion, A. (2004). Increased interhemispheric interaction is associated with decreased false memories in a verbal converging semantic associates paradigm. Brain Cogn. 56, 313-319.

Cukor, J., Olden M., Lee, F., and Difede J. A. (2010). Evidence-based treatments for PTSD, new directions, and special challenges. Ann. N. Y. Acad. Sci. 1208 , 82-89.
Delorme, A., and Makeig, S. (2004). EEGLAB: an open source toolbox for analysis of single-trial EEG dynamics including independent component analysis. J. Neurosci. Methods 134 9-21.

Dionne, H. (1986). Protocol for the Analysis of Interhemispheric Coherence During Paradoxical Sleep. Unpublished master's thesis, University of Montreal, Montreal.

Dummermuth, G., and Lehman, D. (1981). EEG power and coherence during non-REM and REM phases in humans in all-night sleep analyses. Eur. Neurol. 22, 322-339.

Elzinga, B. M., and Bremmer, J. D. (2002). Are the neural substrates of memory the final common pathway in posttraumatic stress disorder (PTSD)? J. Affect. Disord. 70, 1-17.

Essl, M., and Rappelsberger, P. (1998). EEG coherence and reference signals: experimental results and mathematical explanations. Med. Biol. Eng. Comput. 36, 399-406.

Gunter, R. W., and Bodner, G. E. (2008). How eye movements affect unpleas- 
ant memories: support for a workingmemory account. Behav. Res. Ther. 46, 913-931.

Habib, R., Nyberg, L., and Tulving, E. (2003). Hemispheric asymmetries of memory: the HERA model revisited. Trends Cogn. Sci. 7, 241-245.

Hassard, A. (1996). Reverse learning and the physiological basis of eye movement desensitization. Med. Hypotheses 47, 277-282.

Hermans, D., and De Houwer, J. (1994). Affective and subjective familiarity ratings of 740 Dutch words. Psychol. Belg. 34, 115-139.

Jung, T. P., Makeig, S., Humphries, C., Lee, T. W., McKeown, M. J., Iragui, V., and Sejnowski, T. J. (2000). Removing electroencephalographic artifacts by blind source separation. Psychophysiology 37, 163-178.

Kavanagh, D. J., Freese, S., Andrade, J., and May, J. (2001). Effects of visuospatial tasks on desensitization to emotive memories. Br. J. Clin. Psychol. 40, 267-280.

Kemps, E., and Tiggemann, M. (2007). Reducing the vividness and emotional impact of distressing autobiographical memories: the importance of modality-specific interference. Memory 15, 412-422.

Klimesch, W., Sauseng, P., and Hanslmayr, S. (2007). EEG alpha oscillations: the inhibition-timing hypothesis. Brain Res. Rev. 53, 63-88.

Knyazeva, M. G., Kiper, D. C., Vildavski, V. Y., Despland, P. A., Maeder-Ingvar, M., and Innocenti, G. M. (1999). Visual stimulus-dependent changes in interhemispheric EEG coherence in humans. J. Neurophysiol. 82, 3095-3107.

Lamprecht, F., Köhnke, C., Lempa, W., Sack, M., Matzke, M., and Münte, T. F. (2004). Event-related potentials and EMDR treatment of post-traumatic stress disorder. Neurosci. Res. 49, 267-272.

Lee, W. L., and Drummond, P. D. (2008). Effects of eye movement versus therapist instructions on the processing of distressing memories. JAnxiety Disord. 22, 801-808.

Lilley, S. A., Andrade, J., Turpin, G., SabinFarrell, R., and Holmes, E. A. (2009). Visuospatial working memory interference with recollections of trauma. Br. J. Clin. Psychol. 48, 309-321.

Lyle, K. B., Logan, J. M., and Roediger, H. L. (2008a). Eye movements enhance memory for individuals who are strongly right-handed and harm it for individuals who are not. Psychon. Bull. Rev. 15, 515-520.

Lyle, K. B., Roediger, H. L., and McCabe, D.P. (2008b). Handedness is related to memory via interhemispheric interaction: evidence from paired associate recall and source memory tasks. Neuropsychology 22, 523-530.

Lyle, K. B., and Martin, J. M. (2010) Bilateral saccades increase interhemispheric processing but not interhemispheric interaction: implications for saccade-induced retrieval enhancement. Brain Cogn. 73, 128-134.

Maxfield, L., Melnyk, W. T., and Hayman, C. A. G. (2008). A working memory explanation for the effects of eye movements in EMDR. J. EMDR Pract. Res. 2, 247-261.

McNally, R. J., Lasko, N. B., Macklin, M. L., and Pitman, R. K. (1995). Autobiographical memory disturbance in combat-related posttraumatic stress disorder. Behav. Res. Ther. 33, 619-630.

Montgomery, R. W., and Ayllon, T. (1994) Eye movement desensitization across subjects: subjective and physiological measures of treatment efficacy. J. Behav. Ther. Exp. Psychiatry. 25 217-230.

Nunez, P.L. (2000). Towards a quantitative description of large-scale neocortical dynamic function and EEG. Behav. Brain Sci. 23, 371-398.

Nunez, P. L., and Srinivasan, R. (2006) Electric Fields of the Brain: The Neurophysics of EEG, 2nd Edn. New York: Oxford University Press.

Nunez, P. L., Srinivasan, R., Westdorp, A. F., Wijesinghe, R. S., Tucker, D. M., Silberstein, R. B., and Cadusch, P. J. (1997). EEG coherency I: statistics, reference electrode, volume conduction, Laplacians, cortical imaging, and interpretation at multiple scales. Electroencephalogr. Clin. Neurophysiol. 103, 499-515.

Oldfield, R. (1971). The assessment and analysis of handedness: the Edinburgh inventory. Neuropsychologia 9, 97-113.

Parker, A., Buckley, S., and Dagnall, N. (2009). Reduced misinformation effects following saccadic bilateral eye movements. Brain Cogn. 69, 89-97.

Parker, A., and Dagnall, N. (2007). Effects of bilateral eye movements on gist based false recognition in the DRM paradigm. Brain Cogn. 63, 221-225.

Parker, A., and Dagnall, N. (2010). Effects of handedness and saccadic bilateral eye movements on components of autobiographical recollection. Brain Cogn. 73, 93-101.

Parker, A., Relph, S., and Dagnall, N. (2008). Effects of bilateral eye movements on the retrieval of item, associative and contextual information. Neuropsychology 22, 136-145.

Pfurtscheller, G. (2001). Functional brain imaging based on ERD/ERS. Vision Res. 41, 1257-1260.

Propper, R. E., and Christman, S. D. (2008). Interhemispheric interaction and saccadic horizontal eye movements: implications for episodic memory, EMDR, and PTSD. J. EMDR Pract. Res. 2, 269-281.

Propper, R. E., Christman, S. D., and Phaneuf, K. A. (2005). A mixedhanded advantage in episodic memory: a possible role of interhemispheric interaction. Mem. Cogn. 33 751-757.

Propper, R. E, Pierce, J., Geisler, M. W. Christman, S. D., and Bellorado, N. (2007). Effect of bilateral eye movements on frontal interhemispheric gamma EEG coherence: implications for EMDR therapy. J. Nerv. Ment. Dis 195, 785-788.

Schönfeld, S., and Ehlers, A. (2006). Overgeneral memory extends to pictorial retrieval cues and correlates with cognitive features in posttraumatic stress disorder. Emotion 6, 611-621.

Schubert, S. J.,Lee, C. W., and Drummond P. D. (2011). The efficacy and psychophysiological correlates of dualattention tasks in eye movement desensitization and reprocessing (EMDR). J Anxiety Disord. 25, 1-11.

Servan-Schreiber, D. (2000). Eye movement desensitization and reprocessing: is psychiatry missing the point? Psychiatr. Times 17, 36-40.

Servan-Schreiber, D., Schooler, J., Dew, M. A., Carter, C., and Bartone, P. (2006). Eye movement desensitization and reprocessing for posttraumatic stress disorder: a pilot blinded, randomized study of stimulation type. Psychother. Psychosom. 75, 290-297.

Shapiro, E. (2009). EMDR treatment of recent trauma. J. EMDR Pract. Res. 3 141-151.

Shapiro, F. (1989). Eye movement desensitization: a new treatment for posttraumatic stress disorder. J. Behav. Ther. Exp. Psychiatry 20, 211-217.

Shapiro, F. (2001). Eye Movement Desensitization and Reprocessing: Basic Principles, Protocols and Procedures, 2nd Edn. New York: The Guilford Press.
Shobe, E. R, Ross, N. M., and Fleck, J. I. (2009). Influence of handedness and bilateral eye movements on creativity. Brain Cogn. 71, 204-214.

Squire, L. R., Stark, E. L., and Clark, R. E. (2004). The medial temporal lobe. Annu. Rev. Neurosci. 27, 279-306.

Stickgold, R. (2002). EMDR: a putative neurobiological mechanism of action. J. Clin. Psychol. 58, 61-75.

van den Hout, M. A., Engelhard, I. M., Smeets, M. A. M., Hornsveld, H., Hoogeveen, E., de Heer, E., Toffolo, M. B. J., and Rijkeboer, M. (2010). Counting during recall: taxing of working memory and reduced vividness and emotionality of negative memories. Appl. Cogn. Psychol. 24, 303-311.

van den Hout, M. A., Muris, P. Salemink, E., and Kindt, M. (2001). Autobiographical memories become less vivid and emotional after eye movements. Br. J. Clin. Psychol. 40 121-130.

Wagner, A. D., Shannon, B. J., Kahn, I., and Buckner, R. (2005). Parietal lobe contributions to episodic memory retrieval. Trends Cogn. Sci. 9, 445-453.

Witelson, S. F., and Goldsmith, C. H. (1991). The relationship of hand preference to anatomy of the corpus callosum in men. Brain Res. 545, 175-182.

Conflict of Interest Statement: The authors declare that the research was conducted in the absence of any commercial or financial relationships that could be construed as a potential conflict of interest.

Received: 21 December 2010; paper pending published: 07 January 2011; accepted: 16 February 2011; published online: 09 March 2011.

Citation: Samara Z, Elzinga BM, Slagter HA and Nieuwenhuis S (2011) Do horizontal saccadic eye movements increase interhemispheric coherence? investigation of a hypothesized neural mechanism underlying EMDR. Front. Psychiatry 2:4. doi: 10.3389/ fpsyt.2011.00004

This article was submitted to Frontiers in Neuropsychiatric Imaging and Stimulation a specialty of Frontiers in Psychiatry. Copyright (c) 2011 Samara, Elzinga, Slagter and Nieuwenhuis. This is an open-acces article subject to an exclusive license agreement between the authors and Frontiers Media SA, which permits unrestricted use, distribution, and reproduction in any medium, provided the original authors and source are credited. 